\title{
Design of Multiplier-less Continuously Variable Bandwidth Sharp FIR Filters using Modified Gravitational Search Algorithm
}

\author{
James T.G. \\ Department of Electronics and Communication Engg. \\ National Institute of Technology Calicut, \\ Kerala, India.
}

\author{
Elizabeth Elias \\ Department of Electronics and Communication Engg. \\ National Institute of Technology Calicut, \\ Kerala, India.
}

\begin{abstract}
Variable Bandwidth Filters (VBFs) find applications in software defined radios, trans-multiplexers etc. The key features essential for fractional changes in the bandwidth of a VBF are low complexity, easy re-configurability and fine tuning capability. A VBF which has the capability to reduce the bandwidth has been reported recently. This paper proposes the design of a multiplier-less, sharp VBF based on FRM. It is capable of both bandwidth reduction and enhancement. To make the filter totally multiplier-less, the filter coefficients are synthesized in the Canonic Signed Digit (CSD) format. This is formulated as an optimization problem. Recently, Artificial Bee Colony (ABC) algorithm has been proposed for the discrete optimization of the FRM filter performance. In this paper, Gravitational Search Algorithm (GSA) is deployed to get the optimized filter coefficient values.
\end{abstract}

\section{Keywords}

Variable bandwidth filter, Frequency-response masking, Finite impulse response filter, Re-sampling, Artificial Bee Colony Algorithm, Gravitational Search Algorithm, Canonic Signed Digit, Multiplier-less.

\section{INTRODUCTION}

Finite Impulse Response (FIR) filters are widely acceptable in many areas of signal processing and communication due to their exact linear phase and high stability under certain conditions. FIR filters with variable frequency characteristics also have wide range of applications like software defined radio (SDR) channelizers, trans-multiplexers etc. which require online tuning of frequency characteristics and reconfigurability. In the design of a variable FIR filter, we should preserve the finite length and linear phase of the FIR filter for any desired frequency characteristics. For this, high priority should be given for the optimal design of the filter. For the desired spectral characteristics, the design and the updating routine should be simple and the filter should be suitable for real time applications.

The methods for designing variable digital filters can generally be classified into two types: transformation and spectral parameter approximation. In the case of transformation method, a prototype filter with certain frequency characteristics is first designed and the selected transformation is then applied for obtaining the variable cut off frequencies. For the spectral parameter approximation method, the variable bandwidth filter is assumed to be a weighted combination of fixed coefficient filters, and the weights are directly proportional to the spectral parameters of the variable bandwidth filter.

The methods based on transformation [1]-[3], change the length of the filter and require real time computations to find the new filter coefficients. The methods based on spectral parameter approximation [4]-[6] also require real time calculations to find the new impulse response of the overall filter system using the fixed impulse response of sub filters.

A new method based on changing the sampling frequency of the input signal, which will allow continuous variation of the bandwidth of a FIR filter without changing its coefficients and length is discussed in [7]. This is accomplished by interpolating the input series to obtain an intermediate time series at a new sample rate proportional to the required bandwidth. The interpolated series are processed by the fixed length FIR filter now operating at the changed sample rate. The output of the filtered time series is then interpolated back to the original input sample rate. This effectively changes the bandwidth. The complexity reductions as well as the sharp transition bandwidth are achieved by using a FRM based FIR filter which is having large number of zero valued multiplier coefficients [8].

This paper proposes a further reduction in complexity and power consumption by realizing the filter without multipliers. This is achieved by converting the maximum precision filter coefficients into finite precision coefficients using signed power of two (SPT) systems. The SPT system allows the multiplications to be replaced by shift and add operations [9], which reduces circuit complexity. Hence low power hardware realization is possible, because the multipliers are the major power and silicon area consuming components in the physical realizations of filters. CSD representation is a special case of the SPT system which uses both additions and subtractions [10], and this will give minimum number of SPT terms with precision.

Direct rounding of the filter coefficients to the CSD format with restricted number of SPT terms, can result in the deterioration of the filter performance. This necessitates the use of suitable optimization techniques to improve the performance of the restricted CSD represented filters. The classical gradient based optimization techniques cannot be directly applied, because here, the search space consists of integers. Meta-heuristic algorithm is a good choice for getting the optimized performance. Proper selection of the tuning parameters can lead to a global solution to the particular design problem. 
Genetic algorithms (GA) have been established as a good alternative for the optimization of multimodal, multivariable problems. This is a population based evolutionary algorithm where, in each iteration, candidate solutions are generated using Genetic operations like reproduction, crossover and mutation. A binary coded GA has been used for the design of CSD based FRM filter by Yu et.al. [11]. Even though the performance of the optimization technique is good, the convergence speed is low. To improve the performance of the GA, a hybrid scheme was proposed in [12] which combined the GA and Oscillation Search (OS) algorithm. Here, though the convergence is fast, it uses ternary encoding of the filter coefficients. This calls for the use of some restoration techniques to avoid non canonic CSD coefficients and hence results in more computational load. This drawback is avoided in the novel GA reported by Mercier.et.al [12] where the chromosome is formed by binary encoding of the FRM filter coefficients using a look up table approach. This method is capable of obtaining good performance in the frequency domain, but the running time required for the optimization is very large.

So a search for better alternatives for the design of multiplierless FRM filter to be used as a VBF, has resulted in this work. For this, modern meta-heuristic algorithms like Artificial Bee Colony algorithm [13] and Gravitational Search algorithm [14] have been used. The reduced computation time and the non usage of restoration algorithms are the key features of the proposed approaches. Comparative study of the fixed length fixed bandwidth FRM based FIR filter in terms of optimization parameters and performance parameters are compared.

The paper is organized as follows. Section 2 gives a review of the variable bandwidth filter. Section 3 gives an overview of the frequency response masking technique. In Section 4, the CSD representation is briefed. Section 5 gives a brief overview of the meta- heuristic algorithms. The design of the continuous coefficient FRM filter is given in Section 6. The statement of the problem is done in Section 7. Section 8 illustrates the optimized design of multiplier-less FRM filter using the meta-heuristic algorithms ABC and GSA. Section 9 is concerned with the results and analysis of the optimized FRM filter. Variable bandwidth filter using optimized multiplier-less FRM filter is presented in Section 10 and Section 11 concludes the paper.

\section{Review of Variable Bandwidth Filter}

In the case of constant form factor FIR filter, the length of the filter is inversely proportional to the transition bandwidth, which is proportional to the bandwidth of the filter. Thus if the bandwidth of the filter is changed by a factor, the length of the filter is also changed by the same factor.

In order to realize a variable bandwidth filter without changing the number of coefficients or coefficient values, we can utilize the relationship between the sample rate and bandwidth [7]. This can be further elaborated to mean that the interval between the main lobe peak and the first zero crossing of the impulse response is the reciprocal of the filter bandwidth. Also the interval between the samples is the reciprocal of the sample rate. The number of taps of the filter $\left(T_{P}\right)$ is given by $T_{P}=f s / \Delta f$, where ' $f s$ ' is the sampling frequency and ' $\Delta \mathrm{f}$ ' is the transition bandwidth. If the bandwidth is changed, the transition bandwidth is also changed by the same factor. Hence if the number of taps of the filter is fixed, one method of changing the bandwidth is to change the sampling rate. So we can change the absolute bandwidth of a filter by operating it at a different sampling rate. The technique used in [8] is used for changing the bandwidth continuously in decreasing and increasing fashion.

The implementation of the process is represented in Figure 1. An input signal which is initially oversampled is applied to an arbitrary sample rate converter (up or down). The modified signal is processed by the fixed length, fixed bandwidth FRM based FIR filter and the output of the filter is then converted back to the original input sampling rate by using another arbitrary sample rate converter (down or up). This effectively changes the bandwidth.

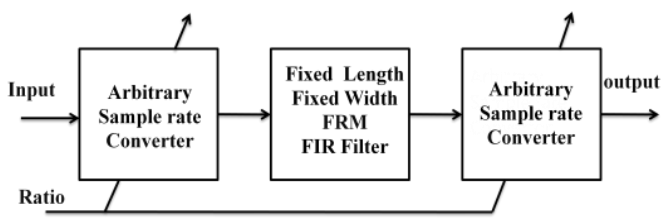

\section{Fig. 1 Functional block diagram of variable} bandwidth filter

Figure 2 shows the implementation of an arbitrary sample rate converter [15]. Three $\mathrm{M}$ - path polyphase filters are used for calculating the sample values of the interpolant and the sample derivatives at the offset position $\mathrm{k} / \mathrm{M}$ from the interpolating output phase centre. The computed output is formed from a local Taylor series as given by Equation (1).

$$
\begin{aligned}
& y\left(n+\frac{k}{M}+\frac{\delta}{M}\right) \cong \\
& y\left(n+\frac{k}{M}\right)+\delta \dot{~}\left(n+\frac{k}{M}\right)+\frac{\delta^{2}}{2 !} \ddot{y}\left(n+\frac{k}{M}\right)+\cdots
\end{aligned}
$$

The increment $d_{\_}$acc satisfies the following relation shown in Equation (2) and the required bandwidth can be achieved by just changing the parameter d_acc as shown in Equation (3).

$$
\begin{aligned}
& \frac{d_{\_} a c c}{M}=\frac{T_{\text {out }}}{T_{\text {in }}}=\frac{f s_{\text {in }}}{f s_{\text {out }}}=\text { Arbitrary sampling ratio } \\
& d_{-} \text {acc }=M * \text { sampling }(\text { bandwidth }) \text { ratio } \\
& \text { Where } \quad M-\text { number of polyphase filters } \\
& f_{s_{i n}} \text { - input sampling rate to interpolate } \\
& \mathrm{fs}_{\text {out }} \text { - output sampling rate of interpolator }
\end{aligned}
$$

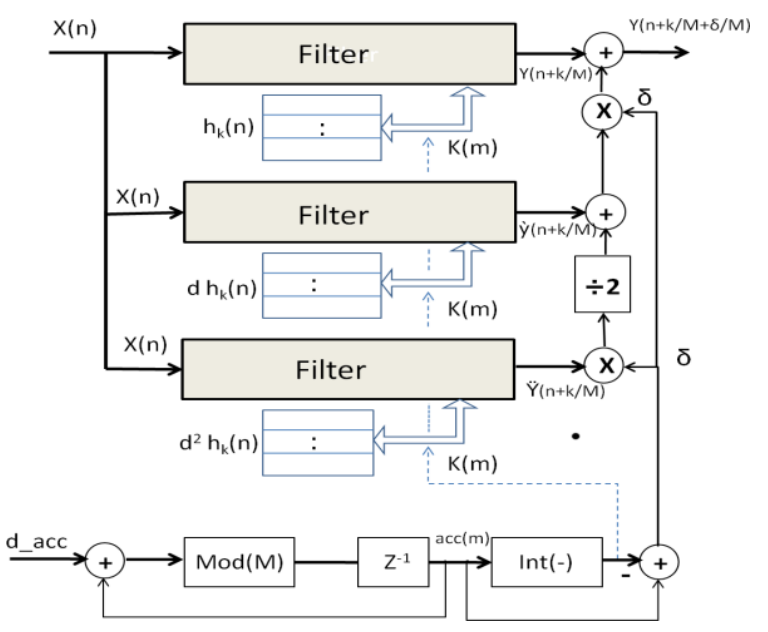

Fig. 2 Implementation of arbitrary sampling rate converter 


\section{Overview of FRM Technique}

In this section, we provide a brief review of FRM technique [16],[17], which is one of the most efficient techniques for the synthesis of sharp linear phase digital filters using several wide transition band sub filters. Compared to the conventional direct-form realization, it gives a large reduction in the computational complexity because of the large number of sparse coefficients.

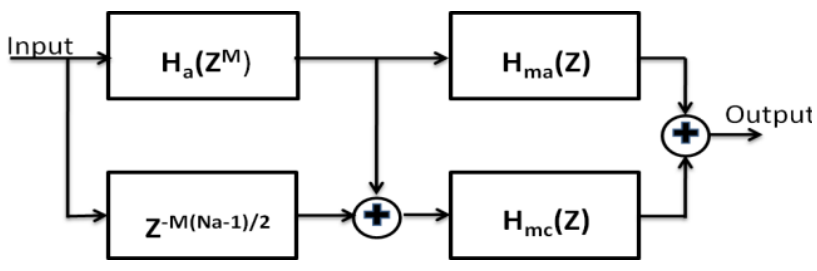

Fig. 3 Realization structure of FRM filter

Figure 3 shows the basic realization structure of the FRM technique. $\mathrm{H}_{\mathrm{a}}\left(\mathrm{z}^{\mathrm{M}}\right)$ is an $\mathrm{M}$ - fold version of a given prototype low pass filter $\mathrm{H}(\mathrm{z})$ with symmetrical impulse response and linear phase. ' $\theta$ ' and ' $\varphi$ ' are the pass band and stop band edges respectively and the transition bandwidth of $\mathrm{H}_{\mathrm{a}}$ $\left(z^{\mathrm{M}}\right)$ is $(\varphi-\theta) / \mathrm{M}$, which is a factor of $\mathrm{M}$ narrower than the transition width of the prototype filter. The complementary filter of $\mathrm{H}_{\mathrm{a}}\left(\mathrm{z}^{\mathrm{M}}\right)$, denoted by $\mathrm{H}_{\mathrm{c}}\left(\mathrm{z}^{\mathrm{M}}\right)$, can be expressed as $\mathrm{H}_{\mathrm{c}}\left(\mathrm{z}^{\mathrm{M}}\right)=\mathrm{z}^{-\mathrm{M}(\mathrm{Na}-1) / 2}-\mathrm{H}_{\mathrm{a}}\left(\mathrm{z}^{\mathrm{M}}\right)$, where $\mathrm{N}_{\mathrm{a}}$ is the length of the impulse response of $\mathrm{H}_{\mathrm{a}}\left(\mathrm{z}^{\mathrm{M}}\right)$ which has to be odd. Two masking filters $\mathrm{H}_{\mathrm{ma}}(\mathrm{z})$ and $\mathrm{H}_{\mathrm{mc}}(\mathrm{z})$ are cascaded to $\mathrm{H}_{\mathrm{a}}\left(\mathrm{z}^{\mathrm{M}}\right)$ and $\mathrm{H}_{\mathrm{c}}\left(\mathrm{z}^{\mathrm{M}}\right)$ respectively and added together to form the FRM filter with an overall transfer function as given in Equation (4). The FRM based filter responses are illustrated in Figure 4 .

$$
\mathrm{H}(\mathrm{z})=\mathrm{H}_{\mathrm{ma}}(\mathrm{z}) \cdot \mathrm{H}_{\mathrm{a}}\left(\mathrm{z}^{\mathrm{M}}\right)+\mathrm{H}_{\mathrm{mc}}(\mathrm{z}) \cdot \mathrm{H}_{\mathrm{c}}\left(\mathrm{z}^{\mathrm{M}}\right)
$$

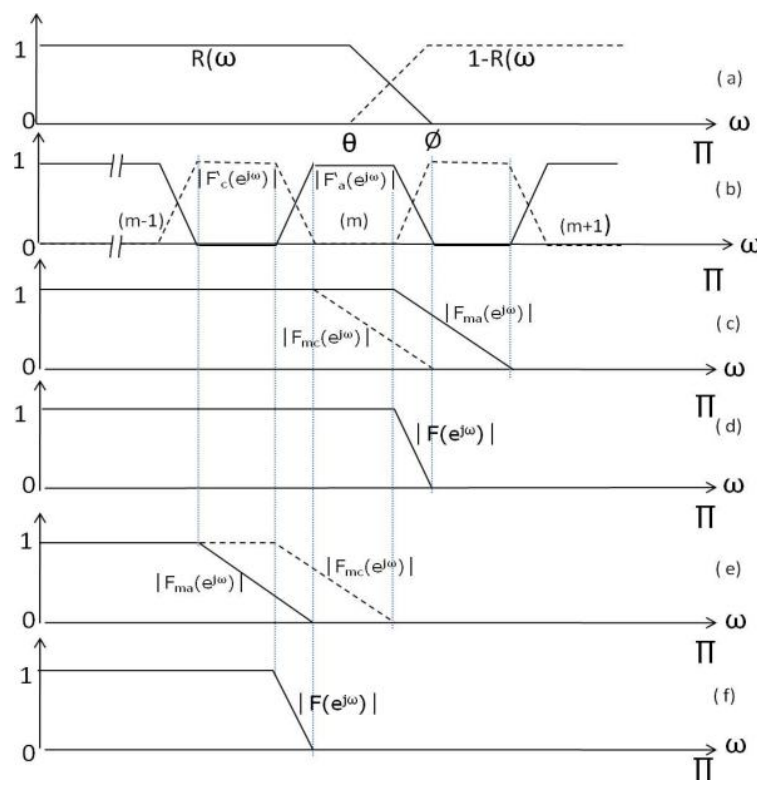

Fig. 4 Illustration of FRM approach

\section{Canonic Signed Digit Representation}

The multipliers are the most power consuming circuits and occupy large silicon area. If it is possible to represent the multiplication coefficients as SPT terms, then the multiplier circuits can be implemented by using shifters and adders/subtraction circuits. CSD representation is an encoding where a binary number contains the fewest number of nonzero bits [9]. The number of partial product addition and hence adders are determined by the total number of non zero bits in the filter coefficient representation. It is possible to represent a filter coefficient with minimum number of SPT terms using CSD representation [12]. A filter coefficient ' $q$ ' can be uniquely represented in CSD format as (5).

$$
\mathrm{q}=\sum_{i=1}^{w} d_{i} 2^{R-i}
$$

where $d_{i} \in\{-1,0,1\}$ called ternary coding and $d_{i} d_{\mathrm{i}-1}=0$. $w$ is the word length of the CSD number $d_{1}, d_{2}, d_{3,-}-d_{w}$. R determines the SPT terms used in CSD numbers. The canonic property $d_{i} d_{\mathrm{i}-1}=0$, ensures that all non-zero digits in the CSD numbers are separated by at least one zero digit. The maximum number of non zero bits in the CSD representation of a $w$ bit number is $(w+1) / 2$ and the maximum number of adder / subtraction needed to realize the CSD represented $w$ bit number is only $(w+1) / 2-1$.

\section{Overview of meta-heuristic optimization algorithms}

Heuristic algorithms are typically intended to find a good solution to an optimization problem by 'trial-and-error' in a reasonable amount of time. Meta-heuristics are considered as higher level techniques or strategies which are intended to combine lower level techniques and tactics for exploration and exploitation of the huge space for parameter search. The population based algorithms $\mathrm{ABC}$ and GSA, which are used in this work, come under this category.

There are two important components in modern metaheuristics, and they are: intensification (exploitation) and diversification (exploration) [18]. For an algorithm to be efficient and effective, it must be able to generate a diverse range of solutions including the potentially optimal solutions, so as to explore the whole search space effectively, while it intensifies its search around the neibourhood of an optimal or near optimal solution. In order to do so, every part of the search space must be accessible though not necessarily visited during search. Diversification is often in the form of randomization with a random component attached to a deterministic component in order to explore the search space effectively and efficiently, while intensification is the exploitation of past solutions so as to select the potentially good solution via elitism or use of memory or both. Any successful meta-heuristic algorithm requires a good balance of the above two components. Typically, the solution starts with some randomly generated, or educated guess and gradually reduces their diversification while increases their intensification at the same time, though how quick to do so is an important issue. By proper selection of the optimization parameters, these algorithms will result in a global solution and the same can be used for other engineering applications also. The heuristic algorithms do not require the objective function to be derivable or even continuous and can be employed directly on the fitness function to be optimized. The following gives an overview about the meta-heuristic algorithms used. 


\subsection{Artificial Bee Colony Algorithm}

Artificial Bee Colony (ABC) Algorithm is a population based meta-heuristic optimization algorithm based on the intelligent behaviour of honey bee foraging. This model was introduced by Karaboga in 2005 and is based on inspecting the behaviours of real bees on finding nectar amounts and sharing the information of food sources to the other bees in the hive. The specialized bees try to maximize the nectar amount stored in the hive by performing efficient division of labour and selforganisation [13 ].

The colony of artificial bees contains three groups of bees: employed bees, onlookers and scouts. The employed bees are associated with specific food sources, onlooker bees watching the dance of employed bees within the hive to choose a food source, and scout bees searching for food source randomly [13]. The onlooker bees and the scout bees are unemployed bees. Initially the scout bees discover the position of all food sources, thereafter the job of employed bee starts. An artificial employed bee probabilistically obtains some modifications on the position in its memory to target a new food source. Later the onlooker bee evaluates the information taken from all employed bees and then chooses a final food source with the highest probability related to its nectar quality. If the fitness value of the new one is higher than that of the previous one, the bee forgets the old one and memorizes the new position. This is called greedy selection. Then the employed bee whose food source has been exhausted becomes a scout bee to search for further food source once again.

In $\mathrm{ABC}$, the positions of food sources represent the possible solutions and the nectar quality of the food source corresponds to the fitness of the associated solution. The onlookers and employed bees perform the exploitation process in the search space, the scouts carry out the exploration process. The number of employed and onlooker bees are the same, and the number is equal to the number of food sources which is equal to the number of solution vectors in the population. Once the searches for better solutions by all the employed bees are completed, the onlooker bees search for better solutions. Employed bees whose solutions cannot be improved through a predetermined number of trials, specified by the user, called "limit", become scouts and their solutions are abandoned. The scout randomly searches for the location of a new food source. When it finds a new food source, it again becomes an employed bee associated with that food source. When all the scouts become employed bees, the position of the best food source found out so far is updated and another iteration of $\mathrm{ABC}$ algorithm begins. The whole process is repeated again and again till the termination condition is satisfied.

The main steps of the algorithm are

1. Initialize the parameters and food source positions.

2. Place the employed bees on their food source and exploit the better source.

3. Place the onlooker bees on the food sources depending on their nectar amount.

4. Determine the sources to be abandoned and send the scouts to the search area for discovering new food sources.

5. Memorize the best food source found so far.

6. Repeat steps 2-5 until the stopping criterion is met.

\subsection{Gravitational Search Algorithm (GSA)}

Gravitational search algorithm is a newly developed population based heuristic optimization method based on the law of gravity and mass interactions [14]. In this approach, the search agents are a collection of masses which interact with each other based on the Newtonian gravity, "Every particle in the universe attracts every other particle with a force that is directly proportional to the product of their masses and inversely proportional to the square of the distance between them" and the law of motion " The current velocity of any mass is equal to the sum of the fraction of its previous velocity and the acceleration, and the acceleration of any mass is equal to the force acted on the system divided by the mass of inertia". Each agent/object has four specifications: position of the masses, inertial mass, active gravitational mass and passive gravitational mass. The position of the masses constitutes the solution space and the performance of each solution is measured in terms of their masses using fitness function. All of the objects attract each other by the gravity force, while this force causes a global movement, all objects towards the objects with heavier mass, which corresponds to the optimum solution to the problem- move more slowly than the lighter ones; this guarantees the exploitation step of the algorithm. Each mass represents a solution and the algorithm is navigated by properly adjusting the gravitational and inertial masses. The position of the agents is updated with every iteration. The algorithm terminates when a fixed amount of iteration is reached. Once the algorithm terminates, the best fitness at the final iteration is taken and the position of the mass of the corresponding agent becomes the global solution of the problem.

The main steps of the proposed algorithm are

1. Search space identification and randomized initialization.

2. Fitness evaluation of agents

3. Update gravitational constant, inertial and total masses, best and worst fitness values etc.

4. Calculation of total force in different directions.

5. Calculation of the acceleration of agents.

6. Updating agent's position and velocity.

7. Repeat steps $3-6$ until the stop criterion is met.

\section{Design example}

\subsection{Design of a continuous coefficient FRM filter}

Consider a typical example for designing a sharp, arbitrary bandwidth FIR filter. The design specifications of the low pass FRM filter are shown below.

Pass Band Edge frequency: $0.1 \pi$

Stop band Edge frequency: $0.109 \pi$

Maximum Pass band ripple: $0.1 \mathrm{~dB}$

Minimum stop band attenuation: $40 \mathrm{~dB}$

The band-edges of the various sub-filters $\mathrm{H}_{\mathrm{a}}(\mathrm{z})$, $\mathrm{H}_{\mathrm{ma}}(\mathrm{z})$ and $\mathrm{H}_{\mathrm{mc}}(\mathrm{z})$ are obtained and the optimal interpolation factor is found in such a way that the total complexity is minimum [16]. The total complexity is quantified in terms of the total number of multipliers needed for the realization of the overall FRM FIR filter. The orders of the sub filters meeting the required specifications are 33,33 and 33 respectively for the model, masking and complementary masking filters. The total number of multipliers required for the implementation is only 51 , which is around $60 \%$ less, compared to minimax approach for designing a FIR filter for the same specifications 


\subsection{CSD Rounded FRM FIR filter}

To represent the continuous coefficient FRM filter in CSD space, each filter coefficient should be rounded to the nearest CSD number. In this paper, the word length of the CSD is taken as 14 bits. A typical example of the CSD representation of a decimal value is shown in Table1.

Table 1. Typical example of a CSD represented number

\begin{tabular}{|c|c|c|c|}
\hline Index & $\begin{array}{c}\text { CSD Rounded } \\
\text { Number }\end{array}$ & $\begin{array}{c}\text { Decimal } \\
\text { Equivala } \\
\text { nt }\end{array}$ & $\begin{array}{c}\text { Number } \\
\text { of SPT } \\
\text { Terms }\end{array}$ \\
\hline 5096 & $\begin{array}{c}2^{0}+2^{-2}-2^{-7}+2^{-9}-2^{-12} \\
01010000-10100-1\end{array}$ & 1.2439 & 5 \\
\hline
\end{tabular}

After the quantization of the maximum precision multiplier coefficient values, the resulting FRM based FIR filter in the CSD space with restricted number of SPT terms can result in the deterioration of the frequency response. Table 2 gives the performance comparison of continuous coefficient filter and CSD rounded filter with maximum of $7,4,3,2$, and one SPT terms in each filter coefficient. From the table it can be seen that, the frequency response parameters deteriorate while decreasing the total number of SPT terms. Figure 5 shows the magnitude response of the CSD represented filter with a maximum of 2 SPT terms.

\section{Statement of the Problem}

The requirement is to design an FRM filter in the CSD space with reduced complexity for hardware implementation by replacing the multipliers with shifters and adders. The direct rounding of the continuous FRM filter coefficients into restricted number of SPT terms, leads to the degradation of performance specifications. An optimization technique has to be used for reducing the difference between two. In this context, our aim is to model this as an approximation problem by formulating a new objective function suitable for the various meta-heuristic algorithms.

\subsection{Formulation of the Objective Function}

The objective function is defined as an approximation error after the filter is converted to the CSD space. The new objective function $\mathrm{F}_{1}(\mathbf{x})$ is formulated as the $\mathrm{L}_{2}$ norm of the error of approximation, which is given in Equation (6).

Table 2. Comparison of FRM parameters for different SPT terms

\begin{tabular}{|c|c|c|c|c|c|c|}
\hline $\begin{array}{c}\text { Parameters of } \\
\text { FRM filter }\end{array}$ & Continuous & $\begin{array}{c}\text { CSD rounded } \\
\text { for 7 SPT } \\
\text { terms }\end{array}$ & $\begin{array}{c}\text { CSD rounded } \\
\text { for 4 SPT } \\
\text { terms }\end{array}$ & $\begin{array}{c}\text { CSD rounded } \\
\text { for 3 SPT } \\
\text { terms }\end{array}$ & $\begin{array}{c}\text { CSD rounded } \\
\text { for 2 SPT } \\
\text { terms }\end{array}$ & $\begin{array}{c}\text { CSD rounded } \\
\text { for 1 SPT } \\
\text { term }\end{array}$ \\
\hline $\begin{array}{c}\text { Max. Pass band } \\
\text { ripple (dB) }\end{array}$ & 0.1163 & 0.1191 & 0.1247 & 0.1263 & $\mathbf{0 . 2 4 3 5}$ & 0.8927 \\
\hline $\begin{array}{c}\text { Min. Stop band } \\
\text { attenuation (dB) }\end{array}$ & 38.9967 & 38.2376 & 37.9796 & 36.3035 & $\mathbf{3 0 . 4 8 6 2}$ & 19.5544 \\
\hline SPT terms in Fa & & 97 & 95 & 81 & $\mathbf{6 0}$ & 31 \\
\hline SPT terms in Fma & & 101 & 96 & 83 & $\mathbf{6 0}$ & 31 \\
\hline SPT terms in Fmc & & 96 & 92 & 79 & $\mathbf{5 4}$ & 29 \\
\hline Total SPT terms & & 294 & 283 & 243 & $\mathbf{1 7 4}$ & 91 \\
\hline
\end{tabular}

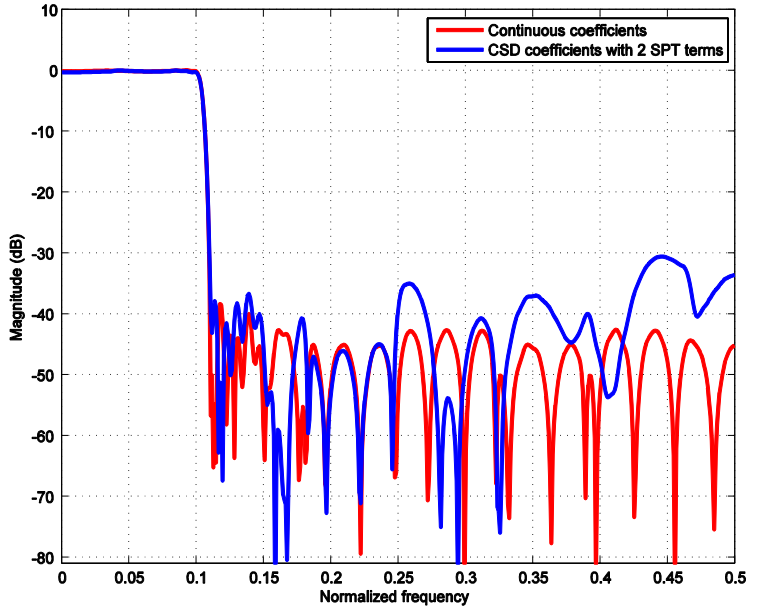

Fig. 5 Magnitude responses of continuous coefficient and CSD

$$
\mathrm{F}_{1}(\mathrm{x})=\left\|\mathrm{H}_{\mathrm{d}}(\omega)-\mathrm{H}(\omega, \mathrm{x})\right\|_{2}
$$

where $H_{d}(\omega)$ is the zero phase frequency response of the maximum precision/ continuous coefficient FRM filter and $H(\omega, x)$ is the zero phase frequency response of the optimized filter, where, $\mathbf{x}$ is the design vector constituted by concatenating the filter coefficients of the sub filters of the FRM filter in the CSD space. To reduce the number of SPT terms in the optimized CSD equivalents, a constraint is also added to the optimization problem as $\mathrm{n}(\mathrm{x}) \leq \mathrm{n}_{\mathrm{b}}$. $\mathrm{n}(\mathrm{x})$ denotes the average number of non zero SPT coefficients after optimization and $\mathrm{n}_{\mathrm{b}}$ is the upper bound of $\mathrm{n}(\mathrm{x})$. The penalty method [19] can be used for keeping the optimization problem as an unconstrained one by including the penalty function given by Equation (7) and the final objective function for the coefficient synthesis of FRM filter can be formulated as Equation (8).

$$
\mathrm{g}(\mathrm{x})=\max \left(0, \mathrm{n}(\mathrm{x})-\mathrm{n}_{\mathrm{b}}\right)
$$

Minimize $\mathrm{F}(\mathbf{x})=\lambda_{1} \mathrm{~F}_{1}(\mathbf{x})+\lambda_{2} \mathrm{~g}(\mathrm{x})$ 
where $\lambda_{1}$ and $\lambda_{2}$ are positive weighting coefficients and can be used for fixing the relative importance of the terms in the objective function of the optimization problem.

\subsection{Encoding of the optimization variables}

For the conversion of the maximum precision FRM filter coefficients into the CSD space, the look up table method is used [20]. The advantages of using look up table method instead of ternary coding are the non usage of restoration algorithm and the reduction in the computational complexity. A CSD look up table with four fields: index, CSD number, decimal equivalent and the number of nonzero SPT terms is created as shown in Table 1 for the entire 14 bit representation, out of which, 12 bits are used for representing the fractional part and 2 bits for the integer part. The decimal value larger than this is limited to the maximum value of representation.

For the joint optimization of the various sub filters, the coefficients of the sub filters $\mathrm{H}_{\mathrm{a}}(\mathrm{z}), \mathrm{H}_{\mathrm{ma}}(\mathrm{z})$ and $\mathrm{H}_{\mathrm{mc}}(\mathrm{z})$, concatenated together, is used to form the design vector for the optimization problem. Since the sub filters are assumed to have linear phase, the number of optimization variables can be further reduced by extracting only half of the symmetrical filter coefficients of each sub filter. This reduces the dimensionality and the computation time compared to the optimization by Mercier et. al. [12].

In order to represent the filter coefficients in the CSD space, they are encoded as the signed indices of the look up table locations of the nearest CSD equivalent. If the decimal filter coefficient is negative, then it is encoded as the negative of the index of the location of its positive counterpart. Thus the initial solution for the joint optimization problem is constituted by concatenating half of the integer coded CSD coefficients of the model filter, masking filter and the complementary masking filter. Since the search space consists of integers, the classical gradient based optimization techniques cannot be used. Therefore the optimization based on meta-heuristic approaches with proper modifications to suit the problem is adopted here.

\section{Design of the Optimal Multiplier-less FRM Filter for Variable Bandwidth using Modified Meta-heuristic Algorithms}

Modern meta-heuristic algorithms such as ABC and GSA are suitable for solving multimodal, multivariable, discrete optimization problems [21]. But ternary coding is not suitable for these algorithms. So in this paper, modified integer coded $\mathrm{ABC}$ and GSA are used for the design of multiplier-less FRM prototype fixed bandwidth filter for the variable bandwidth application. An FRM filter with continuous coefficients is designed and the coefficients of the sub filters are encoded in CSD format. A typical vector in this format is shown in Table 3, where $x a, x m a$ and $x m c$ represent the CSD synthesized coefficients of the model, masking and complementary masking filters respectively. The filter coefficients are then encoded as signed integers using look-up table as explained in Section 7.2. This is used as the initial starting solution vector to the optimization. The maximum number of nonzero bits or SPT terms in a filter coefficient for optimization is taken as 2. The following algorithms are used for the optimized CSD coefficient synthesis.

Table 3. A typical solution vector

$x a_{n 1,-}--x a_{N 1-1}, x m a_{n 2,-}--x m a_{N 2-1}, x m c_{n 3,-}--x m c_{N 3-1}$

\subsection{Design of optimal CSD coefficient filter using integer coded ABC Algorithm}

The various phases of the proposed $\mathrm{ABC}$ algorithm for the CSD coefficient synthesis of FRM filter are explained below [13].

\subsubsection{Initialization}

Population size $N$, maximum number of iteration $M N I$ and the control parameter "limit" are set with typical values given in the Table 4.

Table 4. Typical initialization parameters of integer coded ABC Algorithm

\begin{tabular}{|c|c|c|}
\hline Population size $N$ & limit & MNI \\
\hline 50 & 200 & 500 \\
\hline
\end{tabular}

For ensuring wider search space and elite solutions, at the beginning, the total number of food sources are taken as the integer multiple of the employed bees. The prioritized food sources, which are the same, as the number of employed bees, are selected from the randomly perturbed initial solution based on the nectar quality and passed on to the next stage of the optimization.

\subsubsection{Employed bee phase}

Each employed bee is associated with a food source. For each employed bee determine a new food source within the neighbourhood of the food source in their memory. The new food source is produced by (9).

$$
v_{i j}=\operatorname{round}\left(x_{i j}+\Phi_{i j}\left(x_{i j}-x_{k j}\right)\right)
$$

where $\Phi_{i j}$ is a uniformly distributed real random number in the range $[-1,1], j$ is the random value in the range $(1,2$, $3, \ldots D), k$ is the index of the solution chosen randomly in the range $(1,2,3, \ldots \ldots . N), D$ is the dimension of the solution and $N$ is the number of employed bees.

The new food source is verified for the boundary conditions of the CSD lookup table by Equation (10), where $v_{l b}$ and $v_{u b}$ are the lower bound and upper bound of lookup and $j \in 1,2$, $3 \ldots . . D$ and $i$ is the index of the current solution. The new solution is compared with the existing solution and the employed bee exploits the better source. If the nectar amount or the fitness value is better than the existing one, it is

$$
v_{j i, G+1}=\left\{\begin{array}{lll}
v_{l b} & \text { if } v_{j i, G+1}<v_{l b} \\
v_{u b} & \text { if } v_{j i, G+1}>v_{u b}
\end{array}\right.
$$


replaced with the new food source, otherwise it is retained.

\subsubsection{Onlooker bee phase}

An onlooker bee chooses a food source with a probability $P i$ using (11), which is based on the nectar information collected from all the employed bees and produces a new food source based on (9) as same as in the employed bee phase. Similar to the employed bee phase, the greedy selection mechanism is employed for the selection operation between the old and new food source.

$$
P i=\frac{f i t_{i}}{\sum_{j=1}^{N} f i t_{j}}
$$

where, $f i t_{i}$ is the fitness of the $i_{t h}$ food source and $N$ is the total number of food sources.

\subsubsection{Scout bee phase}

After all the onlookers are distributed to the food sources, the fitness values of the sources are checked whether they are to be abandoned. If the number of cycles that a source cannot be improved is greater than a predetermined "limit", the source is considered to be exhausted. The employed bee associated with the exhausted source become a scout and makes a random search by (12) for finding a new food source. The best food source found so far is memorized.

$$
\mathrm{x}_{\mathrm{t}+1}=\operatorname{randi}([\mathrm{lb}, u b], \text { 'dim' })
$$

where randi denotes the random integer values from the uniform discrete distribution within the interval $[l b, u b]$ with dimension of the food source specified by ' $\mathrm{dim}$ '.

\subsubsection{Termination}

Steps 8.1.2 to 8.1.4 are repeated until the number of predetermined $M N I$ is reached. When the termination condition is met, the solution vector with the best fitness is taken and decoded using lookup table to get the optimal filter coefficients. The magnitude responses of the filters are given in Figure 6.

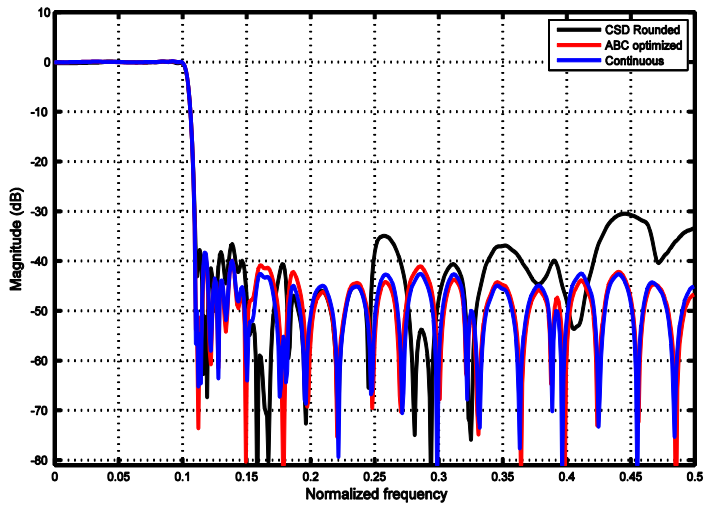

Fig. 6 Magnitude responses of the FRM filter designed using integer coded $\mathrm{ABC}$

\subsection{Design of optimal CSD coefficient filter using integer coded GS Algorithm}

The main steps of the modified GS algorithm for the CSD coefficient synthesis of FRM filter are explained below [14].

\subsubsection{Initialization}

Number of masses (agents) $N$, gravitational constant $G_{0}$, user specified constant $\alpha$ and maximum number of iterations $M N I$ is set with the typical values shown in Table 5 .

Let $N$ number of agents constituted the GSA system. Initialize the position of agents by randomly perturbing the CSD encoded filter coefficient. The position of the $i_{t h}$ agent can be defined by Equation (13).

$\mathrm{X}_{\mathrm{i}}=\left(x_{\mathrm{i}}{ }^{1}, \ldots x_{\mathrm{i}}^{\mathrm{d}}, \ldots x_{\mathrm{i}}^{n}\right)$ for $i=1,2,3, \ldots N$

where $x_{\mathrm{i}}^{\mathrm{d}}$ is the position of the $i_{t h}$ mass in the $d_{t h}$ dimension and $n$ is the dimension of the search space.

Table 5. Typical initialization parameters of integer coded GS Algorithm

\begin{tabular}{|c|c|c|c|}
\hline $\begin{array}{c}\text { Number of } \\
\text { agents } N\end{array}$ & $\begin{array}{c}\text { Gravitational } \\
\text { constant } G_{0}\end{array}$ & $\begin{array}{c}\text { Constant } \\
\alpha\end{array}$ & $M N I$ \\
\hline 50 & 100 & 20 & 500 \\
\hline
\end{tabular}

\subsubsection{Fitness evaluation and updating of}

\section{parameters}

In each iteration the fitness function of the objects are evaluated. After identifying the best and worst values for the current iteration, the masses of the particles are updated according to the following equations. The heavier mass means a more efficient agent which is having more attractions and moves more slowly.

Gravitational and inertial mass of an agent can be calculated using map of fitness.

$$
\begin{gathered}
M_{a i}=M_{p i}=M_{i i}=M_{i}, \quad \text { where } i=1,2,3, \ldots \ldots . . N \\
m_{i}(t)=\frac{f i t_{i}(t)-\operatorname{worst}(t)}{\operatorname{best}(t)-\operatorname{worst}(t)} \\
M_{i}(t)=\frac{m_{i}(t)}{\sum_{j=1}^{N} m_{j}(t)}
\end{gathered}
$$

where, $M_{a i}, M_{p i}$ and $M_{i i}$ are the active gravitational mass, passive gravitational mass and inertia mass respectively of the $i_{t h}$ agent are accepted as they are equal to each other, the $f_{i t}(t)$ represents the fitness value of the agent $i$ at time $t$ and the $\operatorname{best}(t)$ and $\operatorname{worst}(t)$ are the strongest and weakest agent according to their fitness function. Since the optimization problem is a minimization type, the best $(t)$ and $\operatorname{worst}(t)$ can be defined as

$\operatorname{best}(t)=\min _{\mathrm{j} \in 1,2 \ldots N} f i t_{\mathrm{j}}(t) \quad \operatorname{worst}(t)=\max _{\mathrm{j} \in 1,2 \ldots N} f i t_{\mathrm{j}}(t)$

The gravitational constant $G(t)$ is important in determining the performance of GSA. The true value of the gravitational constant is depends on the age of universe and there is a decrease in the gravitational constant with time. Gravitational constant at each iteration can be computed by the Equation (18), the $\mathrm{G}_{0}$ and $\alpha$ were initialized at the beginning, $t$ is the current iteration and $T$ is the maximum number of iteration.

$$
G(t)=\mathrm{G}_{0} e^{-\alpha t / T}
$$

\subsubsection{Calculation of the total forces}

The gravitational force and the total force are updated as follows. $F_{i j}^{d}(t)$ is the gravitational force acting on mass $i$ from mass $j$ in the $d_{t h}$ dimension at time $t$ is defined as 


$$
F_{i j}^{d}(t)=G(t) \frac{M_{p i}(t) M_{a j}(t)}{R_{i j}(t)+\epsilon}\left(x_{j}^{d}(t)-x_{i}^{d}(t)\right)
$$

where, $R_{i j}(t)$ is the Euclidian distance between two objects $i$ and $j$ and $\epsilon$ is a small constant. The total force that act on agent $i$ in the dimension $d$ can be considered as a randomly sum of $d_{t h}$ components of the forces exerted from other agents and is calculated as

$$
F_{i}^{d}(t)=\sum_{j \in K \text { best }, j \neq i}^{N} \operatorname{rand}_{j} F_{i j}^{d}(t)
$$

where, rand $_{j}$ is a random number in the interval $[0,1]$ and Kbest is the set of first $K$ agents with the best fitness value and biggest mass, which is a function of time, initialized to $K_{0}$ at the beginning and decreased with time. Here $K_{0}$ is set to $N$ (total number of agents) and is decreased linearly to 1 .

\subsubsection{Calculation of the acceleration of agents}

According to the law of motion, the acceleration of an agent $i$ at time $t$ in the $d_{t h}$ dimension is given by

$$
a_{i}^{d}(t)=\frac{F_{i}^{d}(t)}{M_{i i}(t)}
$$

where, $M_{i i}(t)$ is the inertial mass of the agent $i$ at time $t$.

\subsubsection{Updating of the position and velocity of the agents}

The next velocity of an agent is a function of its current velocity added to its current acceleration. Therefore the next velocity and the next position of an agent can be calculated by

$$
\begin{gathered}
v_{i}^{d}(t+1)=\operatorname{rand}_{i} v_{i}^{d}(t)+a_{i}^{d}(t) \\
x_{i}^{d}(t+1)=\operatorname{round}\left(x_{i}^{d}(t)+v_{i}^{d}(t+1)\right)
\end{gathered}
$$

where, $x_{i}^{d}, v_{i}^{d}$ and $a_{i}^{d}$ represents the position, velocity and acceleration of the ith agent in the dth dimension respectively. rand $_{i}$ is a uniform random variable in the interval $[0,1]$, which gives a randomized characteristics to the search. The round operation ensures that the candidate solution is an integer.

The new position is verified for the boundary conditions of the CSD lookup table by using Equation (10) in Section 8.1.2 so as to be inside the problem space.

\subsubsection{Termination}

Steps 8.2.2 to 8.2 .5 are repeated until the predetermined maximum number of iterations is reached. When the termination condition is met, the solution vector with the best fitness is taken and decoded using lookup table to get the optimal filter coefficients. The magnitude responses of the filters are given in Figure 7.

\section{Results and analysis of the optimized FRM Filter}

The filter responses resulting from the $\mathrm{ABC}$ and GSA optimization are presented in Figure 8. along with the continuous coefficient and CSD coefficient rounded for two SPT terms.

All the simulations are done using MATLAB 7.10.0 on a Toshiba satellite L750 laptop with Intel (R) core (TM) i5 $2410 \mathrm{M}$ processor operating at $2.3 \mathrm{GHz}$.

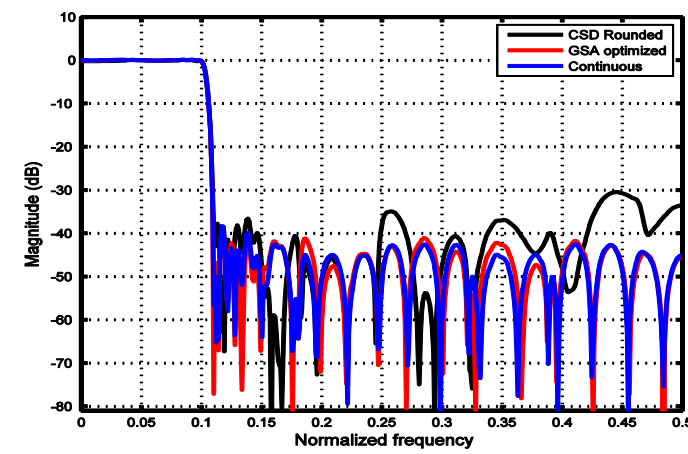

Fig. 7 Magnitude responses of the FRM filter designed using integer coded GSA

The performances of the continuous coefficient, CSD rounded and the optimal FRM filter based on the average of 10 simulations are compared in terms of the pass band ripple and stop band attenuation in Table 6 .

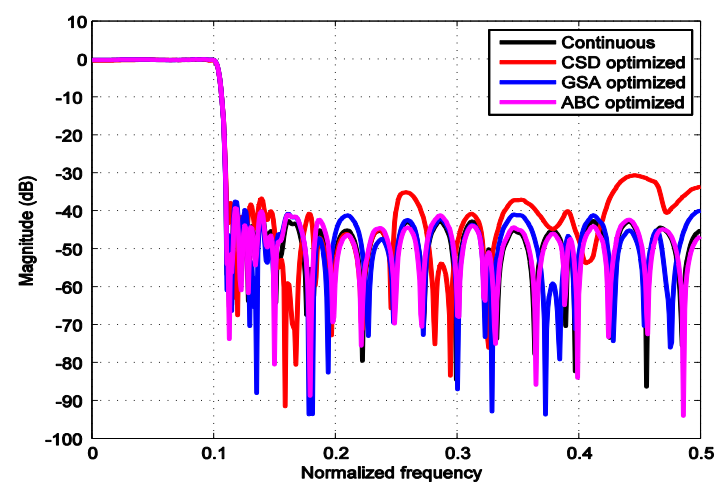

Fig. 8 Magnitude responses of the FRM filter designed using ABC and GSA optimization techniques.

Out of the two different optimization techniques used, taking a total comparison, GSA can be chosen to give the better performance for the given specifications. The GSA gives better performance in terms of complexity and filter performance parameters, even though the run time for the optimization is long. If the run time of optimization is also a criterion for selecting the algorithm, then $\mathrm{ABC}$ algorithm is a better choice.

\section{Variable bandwidth filter using optimized multiplier-less FRM filter}

Variable bandwidth filter with continuous coefficient FRM based filter is presented in [7] which can be used for bandwidth reduction only. In [8], a VBF which is capable of bandwidth reduction as well as enhancement has been proposed by us. The FRM filter in the middle block of the Figure 1 is replaced with the proposed GSA optimized filter and the simulation result of the overall system for the bandwidth reduction and enhancement are given in Figure 9 and Figure 10 respectively. In order to illustrate the working of the variable bandwidth filter using re-sampling, the input signal is formed as a sum of sinusoids spanning the input bandwidth for simulation. Figure 9(a) shows the input spectrum as well as the frequency response of the FRM filter, if the filter is operated at the same sampling rate as that of the input signal. Figure 9(b) is the spectrum of the first sample 
rate converter which is operated as 1 to 0.8 (effective sampling ratio) down sampler. The down sampled but not bandwidth reduced input spectrum is seen expanded. More portions of the signal bandwidth is now seen to extend beyond the filter pass band. Figure 9(c) is the spectrum at the output of the fixed length low pass filter. Figure 9(d) is the spectrum obtained at the output of the second sampling rate converter, which is operated as a 0.8 to 1 up sampler. Hence the input signal is converted back to the original sampling rate. Then the bandwidth is effectively changed. Figure 9(d) also shows the effective bandwidth for visualization.

In order to increase or enhance the bandwidth, the first sampling rate converter in Figure 2 will be operating as an up sampler and the second sampling rate converter will be operating as a down sampler. The corresponding outputs are displayed in Figure 10. The variation of the bandwidth either in the reduction or enhancement in fractional ratio can be achieved by just one parameter change (d_acc) in the arbitrary sample rate converter. Hence the fine tuning of the bandwidth is also possible.
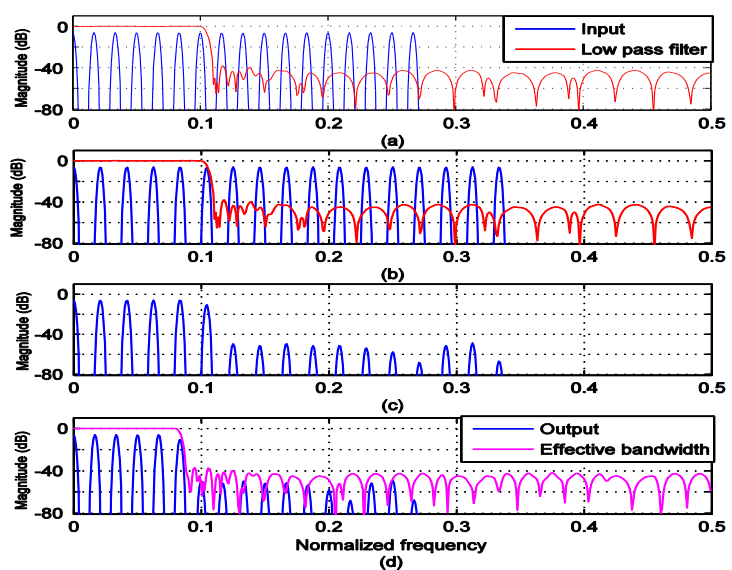

Fig. 9 Input and output of the three processing blocks of Figure 1 with optimized FRM filter for bandwidth reduction.

(a) Spectrum of the input and response of low pass filter. (b)

Spectrum of the output of 1 to 0.8 down-sampler. (c) Spectrum of the output of low pass filter. (d) Spectrum of the output of 0.8 to 1 up-sampler and effective bandwidth.

Table 6. Performance comparison of optimization techniques

\begin{tabular}{|c|c|c|c|c|c|c|}
\hline & \multicolumn{2}{|l|}{ Parameters } & $\begin{array}{l}\text { Continuous } \\
\text { Coefficients }\end{array}$ & $\underset{\text { rounded }}{\text { CSD }}$ & $\mathbf{A B C}$ & GSA \\
\hline \multirow{5}{*}{$\begin{array}{l}\text { Optimization } \\
\text { parameters }\end{array}$} & \multicolumn{2}{|l|}{ Dimension } & & & 51 & 51 \\
\hline & \multicolumn{2}{|l|}{ Population size $N$} & & & 50 & 50 \\
\hline & \multicolumn{2}{|l|}{ Parameter (1) } & & & Limit $=200$ & $\mathrm{G}_{0}=100$ \\
\hline & \multicolumn{2}{|l|}{ Parameter (2) } & & & & $\alpha=20$ \\
\hline & \multicolumn{2}{|c|}{ Max. No. of Iterations $M N I$} & & & 500 & 500 \\
\hline \multirow{7}{*}{$\begin{array}{c}\text { After } \\
\text { optimization }\end{array}$} & \multirow{4}{*}{ No. of SPT terms } & $\mathrm{H}_{\mathrm{a}}$ & & 60 & 49 & 47 \\
\hline & & $\mathrm{H}_{\mathrm{ma}}$ & & 60 & 51 & 50 \\
\hline & & $\mathrm{H}_{\mathrm{mc}}$ & & 54 & 46 & 47 \\
\hline & & Total & & 174 & 146 & 143 \\
\hline & \multicolumn{2}{|c|}{$\%$ saving of SPT terms w.r.t. max. } & & & 50.34 & 51.3 \\
\hline & \multicolumn{2}{|c|}{ Pass band Ripple (dB) } & 0.1163 & 0.2435 & 0.1123 & 0.1076 \\
\hline & \multicolumn{2}{|c|}{ Stop band Attenuation $(\mathrm{dB})$} & 38.9967 & 30.4862 & 39.1721 & 38.9972 \\
\hline \multirow{6}{*}{$\begin{array}{l}\text { Performance } \\
\text { parameters }\end{array}$} & \multicolumn{2}{|l|}{ No. of Multipliers } & 51 & & & \\
\hline & \multicolumn{2}{|l|}{ Coefficient Adders } & 51 & & 51 & 51 \\
\hline & \multicolumn{2}{|c|}{ No. of Adders due to SPT terms } & & & 99 & 95 \\
\hline & \multicolumn{2}{|c|}{ Total number of Adders } & & & 150 & 146 \\
\hline & \multicolumn{2}{|l|}{ Run time (Sec.) } & & & 56.2810 & 136.0258 \\
\hline & \multicolumn{2}{|l|}{ Cost function } & & & $9.6019 \mathrm{e}-06$ & $1.1609 \mathrm{e}-06$ \\
\hline \multirow{7}{*}{$\begin{array}{l}\text { Performance } \\
\text { grading } \\
\text { (numeric) }\end{array}$} & \multicolumn{2}{|l|}{ Run time (Sec.) } & & & 2 & 4 \\
\hline & \multicolumn{2}{|l|}{ No. of SPT terms } & & & 3 & 1 \\
\hline & \multicolumn{2}{|c|}{ Complexity in terms of Adders } & & & 3 & 1 \\
\hline & \multicolumn{2}{|l|}{ Pass band Ripple } & & & 2 & 1 \\
\hline & \multicolumn{2}{|c|}{ Stop band Attenuation } & & & 1 & 3 \\
\hline & \multicolumn{2}{|l|}{ Total } & & & 11 & 10 \\
\hline & \multicolumn{2}{|c|}{ Overall Performance } & & & 2 & 1 \\
\hline
\end{tabular}



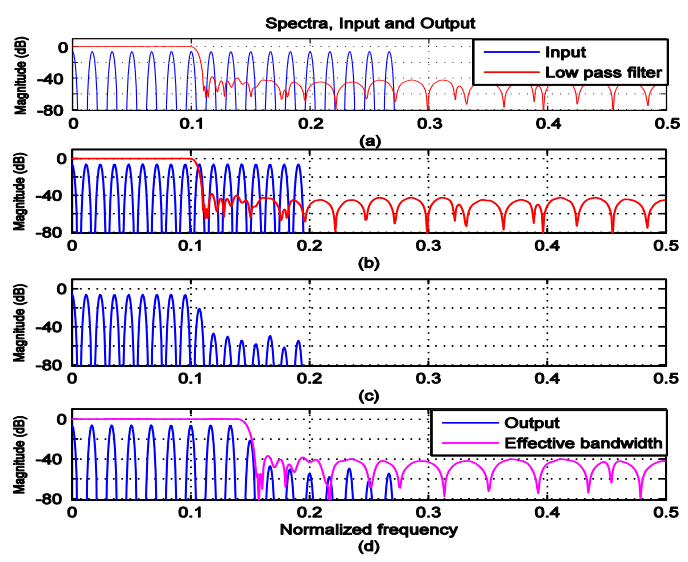

Fig. 10 Input and output of the three processing blocks of Figure 1 with optimized FRM filter for bandwidth enhancement. (a) Spectrum of the input and response of low pass filter. (b) Spectrum of the output of 1 to 1.4 upsampler. (c) Spectrum of the output of low pass filter. (d)

Spectrum of the output of 1.4 to 1 down-sampler and effective bandwidth.

For visualizing the effectiveness of all the optimized filters together with the GSA optimized FRM filter, the variable bandwidth filter is tested with an impulse and the effective filter response in the case of bandwidth reduction and enhancement are shown in Figure 11 and Figure 12 respectively.

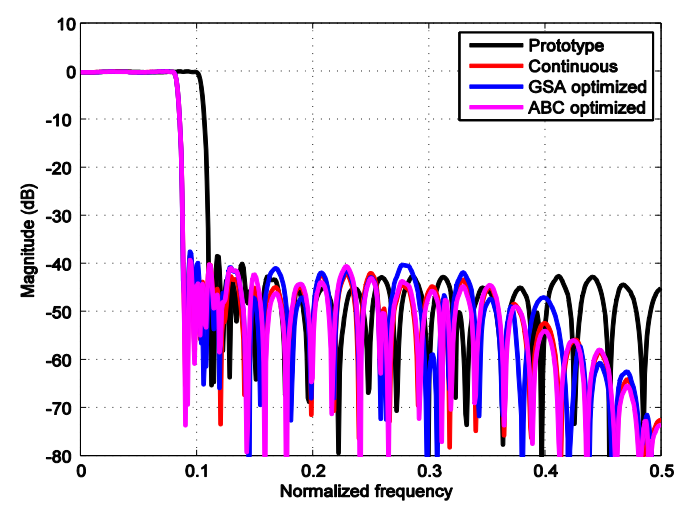

Fig. 11 Effective responses of the variable bandwidth filter using various optimization techniques for a reduction of 0.8 times.

The resulting variable bandwidth filter is computationally efficient and less complex. The reasons for the advantages are

Bandwidth reduction and enhancement are made possible.

- Reduction in complexity is achieved by the use of FRM technique for designing the prototype filter.

- Multiplier-less realization further reduces the complexity and power requirement by replacing the multipliers with shifting and adding/subtraction circuits.

- $\quad$ Easy re-configurability.

- No need of changing the hardware for changing the bandwidth.
- Online computation of filter coefficients is not required.

- Signed integer encoding of the filter coefficient permits the reduction in the running time of the optimization algorithm.

Fine tuning of the bandwidth is possible with fractional change in the bandwidth ratio.

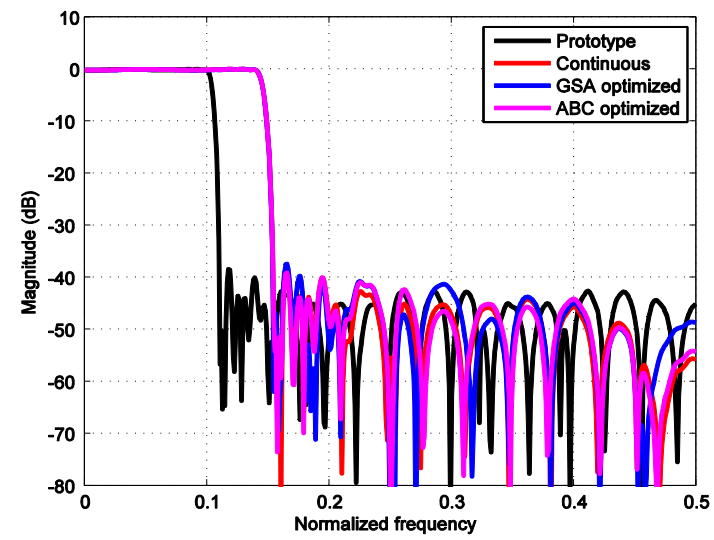

Fig. 12 Effective responses of the variable bandwidth filter using various optimization techniques for an enhancement of 1.4 times.

\section{Conclusion}

In this paper, a technique to obtain a continuously variable bandwidth filter as a combination of two arbitrary sample rate converters and a fixed bandwidth, fixed length, sharp transition bandwidth, low complexity FRM based low pass filter. Further reduction in the complexity is achieved by representing the FRM filter in the CSD space. This reduces the quality of the performance of the system, which demands a proper optimization for optimizing the quality. The design of FRM filter in the CSD space is modelled as an optimization problem. Two different approaches of the modern metaheuristic algorithms ABC and GSA are used for finding the optimized filter representation in the CSD space. Comparison is made in terms of objective function, computation time, number of SPT terms and filter frequency response parameters like pass band ripple and stop band attenuation. The GSA optimization technique gives better performance in terms of complexity and filter performance for this particular design. The GSA optimized FRM filter in the CSD space is used in the variable bandwidth filter and the system performance in the bandwidth reduction and enhancement cases are evaluated. Hence the implementation of the fixed filter in the variable bandwidth application can be done by using only shifters and adder/subtractions. It will reduce the power consumption and silicon area because of the reduction in the total number of multipliers. Hence the continuous variation of bandwidth up to $40 \%$ reduction and $200 \%$ enhancement is achieved with minimum complexity and single parameter modification. Hence easy re-configurability and fine tuning can be achieved.

\section{REFERENCES}

[1] A. V. Oppenheim, W. F.G. Mecklenbrauker, and R. 1 M. Mersereau, 1976, Variable cut off linear phase digital filter, IEEE Transactions on Circuits and Systems, vol. 23, no. 4, pp. 199-203. 
[2] S. Roy and S.S. Ahuja, 1979, Frequency transformations for linear phase variable cutoff digital filters, IEEE Transactions on Circuits and Systems, vol. 26, no. 1, pp. $73-75$.

[3] S.N.Hazra, 1984, Linear phase band pass digital filters with variable cut off frequencies, Circuits and Systems, IEEE Transactions on Circuits and Systems, vol. 31, no. 7, pp. 661-663.

[4] K.S.Pun, S.C.Chan, and K.L.Ho, 2001, Efficient 1d and circular symmetric $2 \mathrm{~d}$ filters with variable cutoff frequencies using the farrow structure and multiplier block, IEEE International Conference on Acoustics, Speech and Signal Processing (ICASSP), pp. 561-562.

[5] Carson.K.S.Pun, S.C.Chan, K.L.Ho, and K.S.Yeung, 2002, On the design and implementation of fir and iir digital filters with variable frequency characteristics, IEEE Transactions on Circuits and Systems, vol. 49, no. 11, pp. 689-703.

[6] H.Johansson and P.Lowenborg, 2004, On linear-phase fir filters with variable bandwidth, IEEE Transactions on Circuits and Systems, vol. 51, no. 4, pp. 181-184.

[7] Fred J. Harris, 2009, Fixed length FIR filters with continuously variable bandwidth, International Conference on Wireless Communication, Vehicular Technology, Information Theory and Aerospace Electronic Systems Technology (Wireless VITAE), IEEE, pp. 931-935.

[8] James T. George and Elizabeth Elias, 2012, Continuously Variable Bandwidth Sharp FIR Filters with Low Complexity, Journal of Signal and Information Processing, vol. 3, pp. 308-315, doi:10.4236/jsip.2012.33040.

[9] Yong Ching Lim D. L., Rui Yang and J. Song, 1999, Signed power-of-two term allocation scheme for the design of digital filters, IEEE Transactions on Circuits and Systems - Analog and Digital Signal Processing, vol. II, pp. 577-584.

[10] Hartley R.I., 1996, Subexpression sharing in filters using canonic signed digit multipliers, IEEE Trans. Circuits Syst. II, vol. 43 , no. 10 , pp. 677-688.
[11] Yu Y.J., Lim Y.C., 2002, Genetic algorithm approach for the optimization of multiplier- less sub-filters generated by the frequency response masking technique, Proceedings of the IEEE International Conference on Electronics, Circuits and Systems, vol.3, pp.1163-1166.

[12] Mercier, P., Mohan-Kilambi, S., Nowrouzian, B., 2007, Optimization of FRM FIR digital filters over CSD and DBNS multiplier coefficient spaces employing a novel genetic algorithm, Computers, (7), 20-31.

[13] Karaboga, D., Basturk, B., 2008, On the performance of artificial bee colony (ABC) algorithm, Appl. Soft Comput. 8 (1), 687-697.

[14] Esmat Rashedi, Hossein Nezamabadi-pour, Saeid Saryazdi, 2009, GSA: A Gravitational Search Algorithm, Information Sciences, Vol. 179, No.13, 2232-2248.

[15] Fred J. Harris, 2004, Multirate Signal Processing for Communication Systems, Prentice Hall.

[16] Y.C. Lim, 1986, Frequency-response masking approach for the synthesis of sharp linear phase digital filters, IEEE Transactions on Circuits and Systems, vol. CAS33, pp. 357-364.

[17] Saramaki, T., Lim, Y.C., 2003, Use of Remez algorithm for designing FRM based FIR filters, Circuits Syst. Signal Process, 22 (2), 77-97.

[18] Xin-She Yang, 2011, Metaheuristic Optimization, Scholarpedia, 6(8):11472.

[19] Boyd S., Vandenberghe L., 2004, Convex Optimization, Cambridge University Press, Cambridge.

[20] Yu Y.J., Y.C. Lim, 2002, A novel genetic algorithm for the design of a signed power-of-two coefficient quadrature mirror filter lattice filter bank, Circuit Syst. Signal Process, 21 (3), 263-276.

[21] Manju Manuel and Elizabeth Elias, 2012, Design of frequency response masking FIR filter in the Canonic Signed Digit space using modified Artificial Bee Colony algorithm, Engineering Applications of Artificial Intelligence, doi:10.1016/j.engappai.2012.02.010 